\title{
Revisiting the cost-effectiveness of universal HPV-vaccination in Denmark accounting for all potentially vaccine preventable HPV-related diseases in males and females
}

Jens Olsen ${ }^{1,2^{*}}$ and Tine Rikke Jørgensen ${ }^{3,4}$

\begin{abstract}
Objective: The purpose of this study was to assess the consequences of a national immunization program with HPV vaccine for both boys and girls in Denmark, including the prophylactic effects on all potentially vaccine preventable HPV-associated diseases in male and female.

Methods: The study focussed on the quadrivalent vaccine which protects against HPV type 6, 11, 16 and 18, and the vaccine's protection against genital warts, cervical intraepithelial neoplasia, cervical cancer, anogenital cancer (anal, penile, vaginal and vulvar cancer) and head and neck cancer (oral cavity, oropharyngeal, hypopharyngeal and laryngeal cancer) were included in the analyses. In general, the analysis was performed in two phases. First, an agent-based transmission model that described the HPV transmission without and with HPV vaccination was applied. Second, an analysis of the incremental costs and effects was performed. The model did not include naturally-acquired immunity to HPV in the simulations.
\end{abstract}

Results: In the base case result (i.e. vaccination of girls only, $85 \%$ vaccination rate, private market price at $€ 123$ per dose ex. VAT) an ICER of 3583 €/QALY (3-dose regime) is estimated when all HPV-related diseases are taken into account. Vaccination of girls \& boys vs. vaccination of girls only an ICER of 28,031 €/QALY (2-dose regime) and 41,636 €/QALY (3-dose regime) is estimated.

Conclusions: Extension of the current HPV programme in Denmark to include boys and girls is a cost effective preventive intervention that would lead to a faster prevention of cancers, cancer precursors and genital warts in men and women.

Keywords: HPV-vaccination, Cost-effectiveness, HPV-related diseases, Cancer, Genital warts, Gender neutral vaccination

\section{Introduction}

Persistent human papillomavirus (HPV) infections with HPV genotype 16 and genotype 18 are responsible for about $70 \%$ of all cervical cancer [1-3]. HPV causes not only cervical cancer, but is also accounting for $40-85 \%$ of cases of anal, penile, vaginal, and vulvar cancer. Not only anogenital cancer is linked to HPV, but also some head and neck cancers, as 16-28\% of cancers of oral cavity,

\footnotetext{
* Correspondence: jo@incentive.dk

${ }^{1}$ Centre for Applied Health Services Research and Technology Assessment (CAST), University of Southern Denmark, 5000 Odense C, Denmark ${ }^{2}$ Incentive, 2840 Holte, Denmark

Full list of author information is available at the end of the article
}

oropharyngeal, hypopharyngeal, and laryngeal are attributable to HPV [4]. HPV 16 and HPV 18 cause most of the HPV linked anogenital cancer and head and neck cancers, as HPV 16 or 18 are accounting for $74-100 \%$ of all these cancers [5-11]. HPV is a cause of not only cancer, the HPV types HPV 6 and 11 cause about $90 \%$ of anogenital warts [12]. In addition juvenile onset of recurrent respiratory papillomatosis is also caused by HPV types 6 and 11 [13].

Two vaccines are currently available on the market: a quadrivalent (including HPV genotypes 16, 18, 6 and 11) and a bivalent vaccine (including genotypes 16 and 18). Both vaccines effectively protect against precancerous 
lesions in the cervix, vulva or vagina and cervical cancer; in addition, the quadrivalent prevents precancerous anal lesions, anal cancer and anogenital warts.

Currently, HPV vaccination of adolescent girls is part of the national immunization programme in a number of countries [14-16]. In the past 5-6 years, numerous cost-effectiveness studies of HPV-vaccination evaluating various vaccination scenarios have been published [14,16-23]. However, few studies include the prophylactic effect of all HPV-associated diseases [18,19,22], despite the considerable burden of non-cervical HPV disease - especially in men $[18,24,25]$. This applies e.g. in Denmark, where the burden of HPV-linked cancer, in particular HPV 16 linked head and neck cancer, is higher in men than in women [25]. In recent years, there has been a moderate decrease in the cervical cancer incidence in Denmark whereas the incidence in head and neck cancer has been increasing. In 2012, the incidence rate (i.e. age adjusted incidence per 100,000) for cervical cancer was 12.7, the incidence rate for anogenital cancer (i.e. anal, penile (in men), vaginal (in women), and vulvar (in women) cancer) was 6.4 in women and 3.6 in men and the incidence rate for head and neck cancer (i.e. cancers of oral cavity, oropharyngeal, hypopharyngeal, and laryngeal) was 8.9 in women and 19.6 in men [26].

Since 2009, the national immunization programme in Denmark offers free quadrivalent human papillomavirus vaccine to all girls aged 12 years. Furthermore, in 2012 and 2013 a catch up program was implemented for all women up to 27 years old.

The purpose of this study was to assess by modelling the economic consequences of a national immunization program with publicly financed quadrivalent HPV vaccine for both boys and girls aged 12 years or a for only girls aged 12 years in Denmark compared with screening program alone, including the prophylactic effects on all $\mathrm{HPV}$-associated diseases.

\section{Methods}

The following scenarios were analysed in the study:

- the cost-effectiveness of vaccinating 12-year-old girls in Denmark compared to no HPV vaccination

and

- the cost-effectiveness of vaccinating 12-year-old girls and boys in Denmark compared to vaccinating girls alone.

The study focussed on the quadrivalent vaccine which protects against HPV type 6, 11, 16 and 18, and the vaccine's protection against genital warts, cervical intraepithelial neoplasia, cervical cancer, anogenital cancer (anal, penile, vaginal and vulvar cancer) and head and neck cancer (oral cavity, oropharyngeal, hypopharyngeal and laryngeal cancer) were included in the analyses. Protection against recurrent respiratory papillomatosis was not included in the analysis among others because no Danish study reports the costs of recurrent respiratory papillomatosis.

\section{Model simulations and assumptions}

A previously developed and published model was used $[15,27]$. This model has also been applied in an Irish setting by the national authorities [28].

The analysis was performed in two phases. First, using the software NetLogo (version 4.0.2) (http://ccl. northwestern.edu/netlogo), an agent based transmission model was developed that describes the HPV transmission dynamics before and after introduction of HPV vaccination. Second, an analysis of the incremental costs and effects was performed using Microsoft $\mathrm{t}^{\bullet}$ Excel. A more comprehensive description can be found in [15] and the original Health Technology Assessment [27] includes an exhaustive description (20 pages) of the model.

In the agent based model the transmission and possible clearance of HPV 6, 11, 16 and 18 was simulated in a heterosexual population and for persistent HPV infections, the subsequent development of genital warts, cervical intraepithelial neoplasia (CIN1-3) and cervical cancer were also simulated. No natural immunity following infection was assumed, suggesting that an individual infected by one type of HPV has the same risk of being re-infected by the same type.

The acquisition of HPV infection and natural course of HPV related cancers at non-cervical sites are poorly understood. Hence, it is difficult to construct a model of HPV infection and disease in these sites that accurately captures the underlying biological processes [29]. As a simplification, it was assumed that the incidence of HPV attributable to non-cervical cancers will decrease at the same rate (proportionately) as cervical cancer, as predicted by the transmission model.

The model operates with several variables, some of which relate to the entire population (e.g. CIN progression and regression probabilities, risk of HPV infection per sexual act) and some of which are agent-specific (e.g. age, sex, duration of relationship), see Table 1 .

It was assumed that the current Danish cervical screening program remained unchanged implying that women aged 23-64 years are offered screening every 3-5 years. Therefore, cervical cancer screening was not modelled. A 62-year time horizon was applied implying that the first year group of 12-year-olds are followed until the age of 74 years in order to include all costs and benefits 
Table 1 Parameters applied in the HPV transmission model

\begin{tabular}{|c|c|c|}
\hline Variable & Value & Source \\
\hline Age groups included & 10-78 years, present Danish age distribution & Age distribution: Statistics Denmark \\
\hline Gender & Man/woman & Fixed at $50 \%$ \\
\hline Concurrent partners & 0,1 or 2, uniform/block distribution & Estimate. \\
\hline Duration of relationship (in months) & $\begin{array}{l}\text { Based on estimate, dependent of age, that is the older the longer } \\
\text { duration }(Y=\text { abs random-normal }(0.8 \text { age }-12)(\text { age/0.5).12). }\end{array}$ & Estimate \\
\hline Frequency of sexual intercourse & $\begin{array}{l}\text { Random-gamma distribution with a mean of } 9.48 \text { per month; } \\
\text { SD } 9.95\end{array}$ & Burchell et al. [30] \\
\hline Vaccination status & 0 or 1 & \\
\hline \multicolumn{3}{|l|}{ HPV-specific: } \\
\hline Duration of HPV 6 infection (in months) & Exponential distribution with a mean of 11 months & \multirow{5}{*}{$\begin{array}{l}\text { The estimate is set in order to calibrate the } \\
\text { model before introduction of a vaccine }\end{array}$} \\
\hline Duration of HPV 11 infection (in months) & Exponential distribution with a mean of 9.5 months & \\
\hline Duration of HPV 16 infection (in months) & Exponential distribution with a mean of 13 months & \\
\hline Duration of HPV 18 infection (in months) & Exponential distribution with a mean of 11 months & \\
\hline $\mathrm{HPV} \rightarrow \mathrm{CIN} 1$ & 0.0049 per month (probability) & \\
\hline $\mathrm{CIN1} \rightarrow \mathrm{HPV} /$ clear (regress) & 0.329 per year (probability) & Elbasha et al. [17] \\
\hline $\mathrm{CIN} 1 \rightarrow \mathrm{CIN} 2$ & 0.46 per year (probability) & \multirow{6}{*}{$\begin{array}{l}\text { The estimate is set in order to calibrate the } \\
\text { model before introduction of a vaccine }\end{array}$} \\
\hline $\mathrm{CIN2} \rightarrow \mathrm{CIN} 1$ (regress) & 0.1 per year (probability) & \\
\hline $\mathrm{CIN} 2 \rightarrow \mathrm{CIN} 3$ & 0.60 per year (probability) & \\
\hline $\mathrm{CIN3} \rightarrow \mathrm{CIN} 2$ (regress) & 0.02 per year (probability) & \\
\hline CIN3 $\rightarrow$ cervical cancer & 0.37 per year (probability) & \\
\hline HPV $6 / 11 \rightarrow$ genital warts & 0.30 per year (probability) & \\
\hline \multicolumn{3}{|l|}{ Risk of infection } \\
\hline -for HPV 6/11/16 per intercourse & 0.3 & Elbasha et al. [17] \\
\hline -for HPV 18 per intercourse & 0.13 & $\begin{array}{l}\text { Modified compared to the HPV } 16 \text { risk to } \\
\text { take into account a lower HPV } 18 \text { prevalence }\end{array}$ \\
\hline Vaccine efficacy & $100 \%$ & \\
\hline
\end{tabular}

of the vaccination programme (i.e. the second year group of 12-year-olds are followed 61 years etc.).

The vaccine's possible protection against non-vaccine HPV types (i.e. cross protection) was not included in the analyses even though slight evidence suggests that some cross protection might exist (SPC Gardasil and Cervarix, http://www.ema.europa.eu/ema). In some studies cross protection are included (e.g. [29]). Thus, cross protection in this analysis implies that equal cross protective efficacy for the two existing vaccines were assumed.

Compared to the previous publication [15], the model has been adjusted with updated data on cancer incidence and mortality.

\section{Cost and effects}

For each scenario the incremental cost (vaccination cost minus future costs averted by vaccination) per life year gained (LYG) and the incremental cost per quality adjusted life year (QALY) gained by the HPV-vaccination was estimated. Given the age distribution for cancer deaths and that for the general population, it was possible to estimate the life-years gained (LYG) due to a lower incidence of cervical cancer, anogenital cancer and head and neck cancer. Similarly, given the modelled decrease in the specific diseases and given the QALY-weights for the general population and the specific diseases (Table 2), the gain in QALYs was estimated.

A health care sector perspective was applied and included direct costs of vaccination and future averted health care sector costs of HPV-associated diseases. The applied health care costs appear from Table 3.

Future costs and effects were discounted at a rate of $3 \%$ in the base case.

\section{Sensitivity analyses}

One-way sensitivity analyses varying one parameter, while holding other parameters at their base case value, were performed. The parameters varied appear from Table 4. Vaccination rate refer to the 3-dose coverage of 12-year-old girls (and boys if applicable).

Based on a recently published study, Danish authorities have changed the quadrivalent vaccine regime from 
Table 2 QALY weights applied

\begin{tabular}{|c|c|c|c|c|c|}
\hline \multicolumn{3}{|c|}{ QALY weights the general population ${ }^{1}$} & & \multirow[b]{2}{*}{ QALY } & \\
\hline & Men & Women & & & \\
\hline Age group & QALY & QALY & Occurrence/illness & Utility score ${ }^{2}$ & Duration \\
\hline $15-19$ & 0.9373 & 0.9203 & & & \\
\hline $20-24$ & 0.9373 & 0.9203 & CIN1 & 0.93 & 2 months \\
\hline $25-29$ & 0.9373 & 0.9203 & & & \\
\hline $30-34$ & 0.9355 & 0.9118 & $\mathrm{CIN} 2-3$ & 0.87 & 2 months \\
\hline $35-39$ & 0.9197 & 0.8898 & & & \\
\hline $40-44$ & 0.9118 & 0.8763 & Genital warts & 0.91 & 85 days \\
\hline $45-49$ & 0.9050 & 0.8751 & & & \\
\hline $50-54$ & 0.8813 & 0.8499 & Cervical cancer, fatal & $0.76-0,67$ & \\
\hline $55-59$ & 0.8870 & 0.8542 & Cervical cancer, survivors & 0.76 & 5 years \\
\hline $60-64$ & 0.8747 & 0.8552 & & & \\
\hline $65-69$ & 0.8801 & 0.8098 & Genital cancer, fatal & $0.76-0.67$ & \\
\hline $70-74$ & 0.8437 & 0.8320 & Genital cancer, survivors & 0.76 & 5 years \\
\hline $75-79$ & 0.8429 & 0.7837 & & & \\
\hline $80-84$ & 0.7855 & 0.6919 & Head \& neck cancer, fatal & $0.76-0.67$ & \\
\hline $85-$ & 0.7855 & 0.6919 & Head \& neck cancer, survivors & 0.76 & 5 years \\
\hline
\end{tabular}

${ }^{1}$ Unpublished data from survey performed by National Institute of Public Health, University of Southern Denmark (personal communication). These data, pooled with two other Danish surveys, are published on a more aggregate level in Sørensen et al., 2009 [31].

${ }^{2}$ Source: [18].

3 doses to 2 doses [32]. Therefore, a sensitivity analysis with a 2-dose regime was also perfomed (cf. Table 4). Furthermore, in order to handle the parameter uncertainty multi-way sensitivity analysis was performed. 10,000 model simulations were conducted and in each simulation the following parameters were randomly assigned to their base case value or an increased/decreased value: vaccination rate $(70 \%$ or $85 \%)$, vaccine price (market price or market price reduced with $25 \%$ ), treatment cost (base case value or reduced with $25 \%$ ), discount rate $(3 \%$ or $5 \%)$ and time horizon (62 years and 40 years). The results of the multi-way sensitivity analysis were reported as cost-effectiveness acceptability curves (Figure 1).

It should be noted that this multi-way sensitivity analysis did not address all sources of uncertainty as some parameters were not included (e.g. duration of relationship, frequency of sexual intercourse). However, these parameters were varied in the NetLogo simulations as distribution for these parameters were assumed (cf. Table 1).

\section{Results}

Simulations before the introduction of vaccination showed that the estimated HPV prevalence replicated the Danish HPV prevalence (data not shown) fairly precise, indicating that the model was well calibrated (cf. [15]). When vaccination was introduced, the prevalence of HPV 6, 11, 16 and 18 decreased and the rate of decrease among others depends on the applied time horizon. Given a 62-year time horizon, the incidence of e.g. cervical cancer is reduced with $74-77 \%$ depending on the vaccination

Table 3 Cost estimates

\begin{tabular}{lll}
\hline & Cost estimate (PV) & Source \\
\hline Vaccination cost & $417 €$ & \\
Treatment costs - genital warts & $247 €$ & Cost estimates in [15] updated to 2008 price level \\
Treatment/control CIN1 and atypia & $34 €$ & \\
Treatment costs CIN2-3 & $2,780 €$ & \\
Treatment costs cervical cancer & $25,546 €$ & Weighted average based on [24] \\
\hline Treatment costs genital cancer & $24,640 €$ & Weighted average based on [25] \\
\hline
\end{tabular}

2008 price level. PV: present value. Applied discount rate: 3\%. 
Table 4 Results: Cost-effectiveness of HPV-vaccination in Denmark including protection against genital cancer and head \& neck cancer

\begin{tabular}{|c|c|c|c|c|c|c|c|c|}
\hline & $\begin{array}{l}\text { Average incremental } \\
\text { vaccination costs } \\
\text { per year }(€, P V)\end{array}$ & $\begin{array}{l}\text { Average savings in } \\
\text { treatment costs } \\
\text { per year }(€, P V)\end{array}$ & $\begin{array}{l}\text { Average incremental } \\
\text { cost per year }(€, P V)\end{array}$ & $\begin{array}{l}\text { Average } \\
\text { LYG (PV) }\end{array}$ & $\begin{array}{l}\text { Average QALYs } \\
\text { gained (PV) }\end{array}$ & $\begin{array}{l}\text { ICER } \\
\text { (€/LYG) }\end{array}$ & $\begin{array}{l}\text { ICER } \\
(€ / Q A L Y)\end{array}$ & $\begin{array}{l}\text { Average number } \\
\text { of cases avoided } \\
\text { per year }\end{array}$ \\
\hline \multicolumn{9}{|l|}{ Vaccinations of girls vs. screening alone: } \\
\hline Base case result* & $11,504,613$ & $9,480,325$ & $2,024,288$ & 528.5 & 565.3 & 3,830 & 3,581 & \\
\hline Cervical cancer & & & & & & & & 197 \\
\hline Anogenital cancer & & & & & & & & 80 \\
\hline Head and neck cancer & & & & & & & & 43 \\
\hline \multicolumn{9}{|l|}{ Univariate sensitivity analysis: } \\
\hline Discount rate $0 \%$ & $11,504,613$ & $19,622,317$ & $-8,117,704$ & $1,706.5$ & $1,645.5$ & & Dominance & Dominance \\
\hline Discount rate $5 \%$ & $11,504,613$ & $6,603,582$ & $4,901,031$ & 265.3 & 312.5 & 18,476 & 15,682 & \\
\hline Costs discounted with $3 \%$ and LYG \& QALYs with $0 \%$ & $11,504,613$ & $9,480,325$ & $2,024,288$ & $1,706.5$ & $1,645.5$ & 1,186 & 1,230 & \\
\hline Vaccine price reduced by $25 \%$ & $8,967,267$ & $9,480,325$ & $-513,058$ & 528.5 & 565.3 & & Dominance & Dominance \\
\hline 2-dose regime & $8,121,485$ & $9,480,325$ & $-1,358,840$ & 528.5 & 565.3 & & Dominance & Dominance \\
\hline Treatment cost reduced by $25 \%$ & $11,504,613$ & $7,055,464$ & $4,449,149$ & 528.5 & 565.3 & 8,418 & 7,870 & \\
\hline Time horizon 40 years & $11,504,613$ & $8,450,127$ & $3,054,486$ & 425.2 & 472.2 & 7,184 & 6,469 & \\
\hline Bivalent HPV vaccination (HPV types 16 \& 18) & $11,504,613$ & $5,269,690$ & $6,234,923$ & 528.5 & 505.1 & 11,797 & 12,345 & \\
\hline Protection against head and neck cancer excluded: & $11,504,613$ & $9,159,308$ & $2,345,305$ & 432.1 & 470.4 & 5,428 & 4,985 & \\
\hline $70 \%$ vaccination rate & $9,474,387$ & $9,320,914$ & 153,473 & 489.2 & 551.9 & 314 & 278 & \\
\hline Cervical cancer & & & & & & & & 192 \\
\hline Anogenital cancer & & & & & & & & 77 \\
\hline Head and neck cancer & & & & & & & & 42 \\
\hline \multicolumn{9}{|l|}{ Vaccination of girls \& boys vs. girls: } \\
\hline Base case result:** & $11,858,601$ & $1,186,760$ & $10,671,841$ & 263 & 256 & 40,615 & 41,636 & \\
\hline Cervical cancer & & & & & & & & 5 \\
\hline Anogenital cancer & & & & & & & & 34 \\
\hline Head and neck cancer & & & & & & & & 98 \\
\hline \multicolumn{9}{|l|}{ Univariate sensitivity analysis: } \\
\hline Discount rate $0 \%$ & $11,858,601$ & $4,298,652$ & $7,559,950$ & 889 & 863 & 8,500 & 8,763 & \\
\hline
\end{tabular}

\begin{abstract}
Discount rate 0\%
\end{abstract}
$4,298,652$

$7,559,950$

$889 \quad 863$

8,500 
Table 4 Results: Cost-effectiveness of HPV-vaccination in Denmark including protection against genital cancer and head \& neck cancer (Continued)

Discount rate $5 \%$

Vaccine price reduced by $25 \%$

2-dose regime

Time horizon 40 years

Protection against head and neck cancer excluded

$70 \%$ vaccination rate

\section{8 price level.}

*Vaccination over 62 years (implying that the incremental costs and effects are calculated as an average over the 62 years, assuming vaccination from the first year and onward) of 12 -year-old girls compared to screening alone, $85 \%$ vaccination rate, discount rate $3 \%, 100 \%$ vaccine efficacy, vaccine price: $€ 123$ per dose ex. VAT (=market price, source www.promedicin.dk, 10th of May, 2012).

**Vaccination over 62 years of 12 -year-old girls and boys compared to 12 -year-old girls alone, $85 \%$ vaccination rate, discount rate $3 \%$, 100\% vaccine efficacy, vaccine price: $€ 123$ per dose ex. VAT (=market price, source

PV: present value; QALY: quality-adjusted life-year; ICER: incremental cost-effectiveness ratio; LYG: life-year gained. 


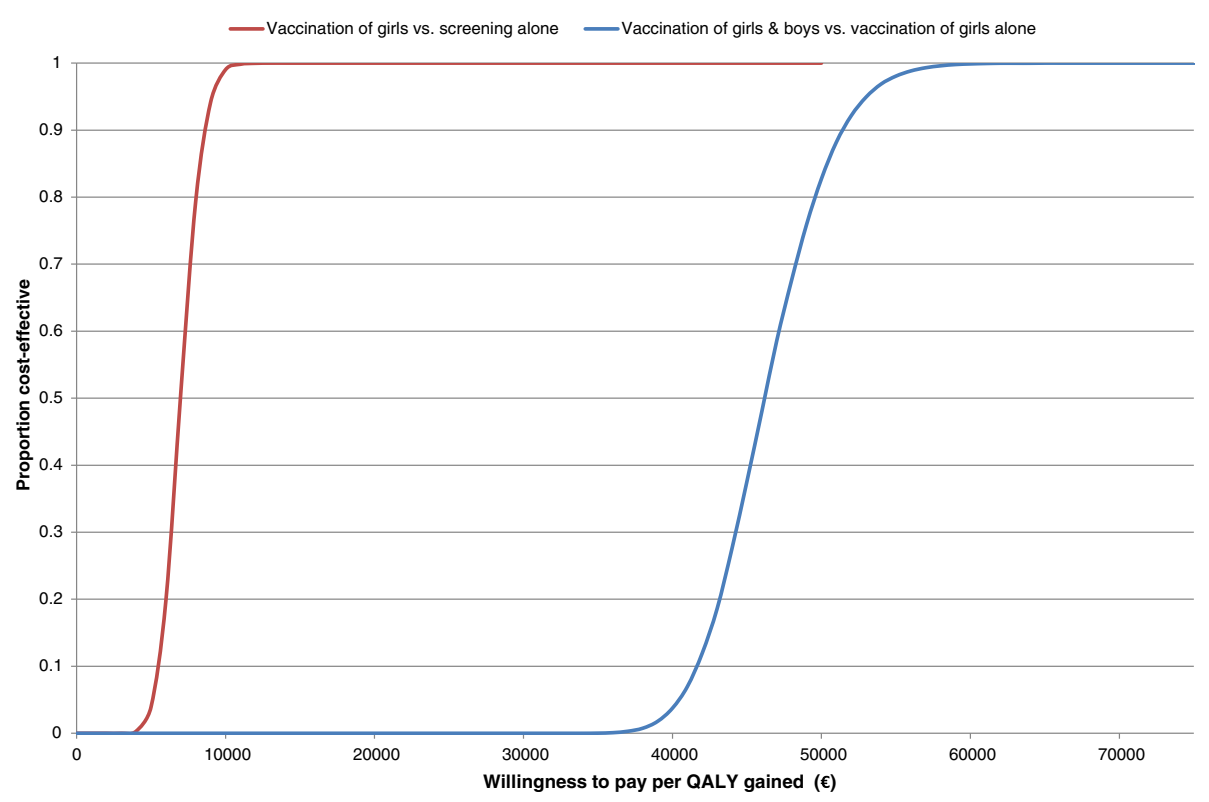

Figure 1 Cost-effectiveness acceptability curves: proportion of simulations that would be cost-effective given different thresholds values of cost per QALY gained. It should be noted that this multi-way sensitivity analysis did not address all sources of uncertainty as some parameters were not included.

program and vaccination rate. A 40-year time horizon leads to an estimated reduction in the incidence of cervical cancer on $59 \%$.

In the base case result with a $85 \%$ vaccination rate the cost per QALY gained by female vaccination (compared to no vaccination) was $€ 3,581$ when including all $\mathrm{HPV}$ related diseases in the analysis (Table 4). A 85\% vaccination rate is the relavant base case as recently updated Danish HPV vaccination rate report coverages on $81-90 \%$ for 3 doses [33]. Compared to this the cost per QALY gained when only protection against cervical cancer and precancerous lesion are included in the analysis is $€ 20,644$ (result not shown in Table 4).

From Table 4 it is seen that the incremental costeffectiveness ratios (ICERs) increases with increasing discount rate, reduced treatment cost, reduced time horizon and exclusion of protection against head and neck cancer whereas the ICERs decreases with reduced discount rate, reduced vaccination rate, reduced vaccine price and application of a 2 -dose regime. Sensitivity analysis on reduced vaccine price is relevant as the vaccine may be offered at a lower price to the health authorities in a public financed programme. Given a $85 \%$ vaccination rate and a $25 \%$ reduction in the vaccine price or application of a 2-dose regime the average annual savings in treatment costs outweigh the average incremental vaccination costs per year, making the vaccination program dominant.

Inclusion of the vaccine's protection against anogenital cancer and head and neck cancer, (the latter being more prevalent in men), in the analysis may better address the relevance of routine vaccination of boys. ICERs of vaccination of girls and boys compared to vaccinaition of girls only appear from Table 4. Compared to vaccination of girls alone the ICERs are higher and again the ICERs increases with increasing discount rate, time horizon and exclusion of protection against head and neck cancer while the ICERs decreases with reduced discount rate, reduced price, application of a 2-dose regime and reduced vaccination rate.

In Denmark, routine vaccination of girls has been conducted since 2008. Currently some countries have expanded the HPV immunization programme to include routine vaccination of boys [34]. Therefore, modelling of universal vaccination of boys and girls compared to vaccination of girls alone may be relevant in a Danish setting. The ICERs are higher implying that it may be beneficial to include boys in the vaccination program as well but the additional effect is decreasing as boys to a certain degree already are protected in a female vaccination program due to herd protection. On the other hand, given a cost-effectiveness threshold on 50,000 $€$ per QALY gained vaccination of boys and girls is estimated to be cost-effective. Vaccinating boys, to prevent diseases that affect women only (cervical, vulvar, and vaginal cancer), lead to relative high ICERs but when all HPV-related diseases are included (penile cancer, anal cancer and head and neck cancer) the ICERs for vaccination of boys and girls versus vaccination of girls alone dropped markedly. 
The uncertainty in the results of the cost-effectiveness analysis is summarized in the cost-effectiveness acceptability curves (Figure 1). In Denmark, there is no defined threshold values of cost per QALY gained but it is seen from Figure 1 that vaccinating girls is cost-effective even at relative low thresholds values.

\section{Discussion}

This study was based on a Danish agent based model on HPV transmission and vaccination. The model was initially developed for the Danish National Board of Health [27]. This study modelled all HPV related diseases in males and females as opposed to previous studies focussing primarily on prevention of HPV related cervical cancers including precursors for cervical cancers. Denmark has a relatively high incidence of HPV related diseases in men and women and potential routine vaccination of girls and boys has continuously been discussed after the publication of a Danish Health Technology Assessment in 2007 [27]. In addition, several countries recommend HPV vaccination of boys as part of the national immunization programme like US, Australia and Canada and some regions in Germany and Austria. In Australia for instance, HPV vaccination of all girls and boys is implemented through a routine school based programme.

In the base case result (i.e. vaccination of girls only, $85 \%$ vaccination rate) an ICER of $3,581 € /$ QALY is estimated when all HPV-related diseases are taken into account. Compared to some studies these are relative low ICERs. However, the results vary with the assumptions and the results are sensitive to the choice of discount rate, the choice of time horizon, vaccine price and vaccination rate. Seen in that perspective, the range of results should be considered. However, these Danish results estimating relative low ICERs is to some extent due to the high Danish incidence and mortality of HPV related cancers (e.g. the incidence and mortality from cervical cancer are among the highest in Europe, cf. Figure 3.3 and 3.4, page 33 in National Board of Health [27]). The incidence of genital warts is also relatively high in Denmark compared with other European countries [35] - resulting in a relatively large savings potential when a quadrivalent HPV vaccination program is introduced (the total treatment cost for genital warts is estimated to 8 mill. $€$ per year [15]).

Comparison with results from others studies analysing $\mathrm{HPV}$-vaccination in other countries is not straightforward because of differences in modelling approach (e.g., dynamic/agent-based versus cohort models) and other parameters, including vaccination scenario (2 or 3 doses) and other country specific conditions. However, the results for female vaccination only lie within the same range/level as the study from Elbasha et al. [18] - with the reservation that Elbasha et al. in principle include a catch-up programme (in the 2010-publication [15] it is shown that at catch-up programme increase the ICERs markedly). When including all HPV-related diseases in an US-setting Elabasha et al. estimate a mean ICER of 3232 \$ per QALY gained in a scenario where girls and women 926 years of age were offered the vaccine [18]. Similarly, Chesson et al. in an US-setting estimated and ICER of 10500 \$ per QALY gained given a 75\% vaccination rate and vaccination of females aged 12-26 years [22].

On the other hand, Kim et al. [19], also in an US-setting, estimate an ICER of 18,130-20,990 \$ per QALY gained in a scenario where 12 -year old girls are vaccinated. The results are very sensitive to price.

Naturally, this analysis has a number of limitations. Firstly, only heterosexual transmission of HPV was simulated ignoring transmission between homosexual and heterosexual persons. Men who have sex with men (MSM) have a higher HPV disease burden (genital warts and anal cancers) and the current HPV programme will not protect this high-risk ground.

Secondly, unlike most other studies this model did not include naturally-acquired immunity to HPV in the simulations.

Thirdly, the model simulates a closed society with no continuous influx of unvaccinated persons. Increased globalisation increases the risk of HPV transmission across national borders thereby lowering the impact of potential the herd protection of men.

Fourth, 100\% vaccine efficacy and lifelong protection was assumed. Assuming a reduced vaccine efficacy (99.8\% or $93.5 \%)$ only change the results on the margin as shown in the 2010-publication [15]. On the other hand, inclusion of one booster dose (i.e. assuming that lifelong protection is not achievable) leads to an increase in the estimated ICERs (results not shown).

Fifth, it was assumed that the current Danish cervical screening program remained unchanged. Presently, the cervical screening program is being reviewed and it may in the long run be modified due to a decrease in the incidence of cervical cancer. Inclusion of a future modification of the screening program, leading to a possible reduction in the screening cost, in principle should be taken into account. However, it has not been taken into account leading to a marginal inaccurate estimation of future costs related to HPV-vaccination. Although sensitivity and scenario analyses are performed, health economic analyses using modelling is a simplified representation of real life involving several assumptions.

Sixth, two different sources were used for the applied QALY weights for the general population and for the specific occurrences/illnesses (Table 2). Furthermore, the QALY weights for the general population were distributed according to age group. This can lead to discrepancies. It 
appears from Table 2, for example, that the utility score for CIN1 was higher than any of the utility scores for the general female population, suggesting that the occurrence of CIN1 will not lead to any QALY loss. This is a limitation of the analysis, primarily caused by the use of two different sources for QALY weights. It is thus possible that the total gain in QALYs is underestimated in the present results.

Finally, possible future variations in the incidence of HPV-related cancers are not incorporated in the model simulations. The overall incidence of HPV related cancers has increased over the last 30 years in Denmark and the incidence of head and neck cancers is expected to increase further in the future. An underestimation of the future incidence leads to an underestimation of the cost of head and neck cancer.

Extension of the current HPV programme in Denmark to include boys and girls is a cost effective preventive intervention that would lead to a faster prevention of cancers, cancer precursors and genital warts in Denmark which is the country in the world with the highest risk of getting a cancer diagnosis [4].

\section{Competing interests}

This study was supported by an unrestricted research grant from Sanofi Pasteur MSD to the Centre for Applied Health Services Research and Technology Assessment, University of Southern Denmark. J. Olsen has received speaker fees from Sanofi Pasteur MSD.

T.R. Jørgensen has been employed by Sanofi Pasteur MSD.

\section{Authors' contributions}

JO developed the updated model based on the original agent based model, designed the study and performed the analyses including sensitivity and scenario analyses and drafted the manuscript. TRJ designed the study, and critically reviewed and revised the manuscript. Both authors read and approved the final manuscript.

\section{Acknowledgement}

Acknowledgement and appreciation to Martin Rudbeck Jepsen, MSc and $\mathrm{PhD}$, who developed the original agent based model published by the National Board of Health in the HTA report on HPV vaccination, and who participated in later modifications of the model. Martin Rudbeck Jepsen has not been a part of this study.

\section{Author details \\ ${ }^{1}$ Centre for Applied Health Services Research and Technology Assessment (CAST), University of Southern Denmark, 5000 Odense C, Denmark. ${ }^{2}$ Incentive, 2840 Holte, Denmark. ${ }^{3}$ Sanofi Pasteur MSD ApS, 2800 Kgs, Lyngby, Denmark. ${ }^{4}$ Medivir, 2800 Kgs, Lyngby, Denmark.}

Received: 28 April 2014 Accepted: 5 January 2015

Published online: 11 February 2015

\section{References}

1. Clifford GM, Rana RK, Franceschi S, Smith JS, Gough G, Pimenta JM. Human papillomavirus genotype distribution in low-grade cervical lesions: comparison by geographic region and with cervical cancer. Cancer Epidemiol Biomarkers Prev. 2005;14:1157-64.

2. Smith JS, Lindsay L, Hoots B, Keys J, Franceschi S, Winer R, et al. Human papillomavirus type distribution in invasive cervical cancer and high-grade cervical lesions: a meta-analysis update. Int J Cancer. 2007;121:621-32.

3. Munoz N, Bosch FX, de Sanjosé S, Herrero R, Castellsague X, Shah KV, et al. Epidemiologic classification of human papillomavirus types associated with cervical cancer. N Engl J Med. 2003;348:518-27.
4. WHO International Agency for Research on Cancer. IARC Monographs on the Evaluation of Carcinogenic Risk to Humans. Human Papillomavirusses. Volume 90. 2007.

5. Parkin DM, Bray F. Chapter 2: the burden of HPV-related cancers. Vaccine. 2006;24 Suppl 3:S3/11-25.

6. Giuliano AR, Tortolero-Luna G, Ferrer E, Burchell AN, de Sanjose S, Kjaer SK, et al. Epidemiology of human papillomavirus infection in men, cancers other than cervical and benign conditions. Vaccine. 2008;26 Suppl 10:K17-28.

7. Hoots BE, Palefsky JM, Pimenta JM, Smith JS. Human papillomavirus type distribution in anal cancer and anal intraepithelial lesions. Int J Cancer. 2009;124:2375-83.

8. Tota JE, Chevarie-Davis M, Richardson LA, Devries M, Franco EL. Epidemiology and burden of HPV infection and related diseases: implications for prevention strategies. Prev Med. 2011;53 Suppl 1:S12-21.

9. Miralles-Guri C, Bruni L, Cubilla AL, Castellsague X, Bosch FX, de Sanjosé S. Human papillomavirus prevalence and type distribution in penile carcinoma. J Clin Pathol. 2009;62:870-8.

10. De VH, Clifford GM, Nascimento MC, Madeleine MM, Franceschi S. Prevalence and type distribution of human papillomavirus in carcinoma and intraepithelial neoplasia of the vulva, vagina and anus: a meta-analysis. Int J Cancer. 2009:124:1626-36.

11. Kreimer AR, Clifford GM, Boyle P, Franceschi S. Human papillomavirus types in head and neck squamous cell carcinomas worldwide: a systematic review. Cancer Epidemiol Biomarkers Prev. 2005;14:467-75.

12. Lacey CJ, Lowndes CM, Shah KV. Chapter 4: Burden and management of non-cancerous HPV-related conditions: HPV-6/11 disease. Vaccine. 2006; 24 Suppl 3:S35-41.

13. Chesson HW, Forhan SE, Gottlieb SL, Markowitz LE. The potential health and economic benefits of preventing recurrent respiratory papillomatosis through quadrivalent human papillomavirus vaccination. Vaccine. 2008;26:4513-8.

14. Jit $\mathrm{M}$, Choi $\mathrm{YH}$, Edmunds WJ. Economic evaluation of human papillomavirus vaccination in the United Kingdom. BMJ. 2008;337:a769.

15. Olsen J, Jepsen MR. Human papillomavirus transmission and costeffectiveness of introducing quadrivalent HPV vaccination in Denmark. Int J Technol Assess Health Care. 2010;26(2):183-91.

16. Kim JJ. Targeted human papillomavirus vaccination of men who have sex with men in the USA: a cost-effectiveness modelling analysis. Lancet Infect Dis. 2010;10:845-52.

17. Elbasha EH, Dasbach EJ, Insinga RP. Model for assessing human papillomavirus vaccination strategies. Emerg Infect Dis. 2007;13:28-41.

18. Elbasha EH, Dasbach EJ. Impact of vaccinating boys and men against HPV in the United States. Vaccine. 2010;28:6858-67.

19. Kim JJ, Goldie SJ. Cost effectiveness analysis of including boys in a human papillomavirus vaccination programme in the United States. BMJ. 2009; 339:b3884.

20. Taira AV, Neukermans CP, Sanders GD. Evaluating human papillomavirus vaccination programs. Emerg Infect Dis. 2004;10:1915-23.

21. Zechmeister I, Blasio BF, Garnett G, Neilson AR, Siebert U. Cost-effectiveness analysis of human papillomavirus-vaccination programs to prevent cervical cancer in Austria. Vaccine. 2009:27:5133-41.

22. Chesson HW, Ekwueme DU, Saraiya M, Dunne EF, Markowitz LE. The cost-effectiveness of male HPV vaccination in the United States. Vaccine. 2011;29:8443-50.

23. Chesson HW, Ekwueme DU, Saraiya M, Watson M, Lowy DR, Markowitz LE. Estimates of the annual direct medical costs of the prevention and treatment of disease associated with human papillomavirus in the United States. Vaccine. 2012;30:6016-9.

24. Olsen J, Jørgensen TR, Kofoed K, Larsen HK. Incidence and cost of anal, penile, vaginal and vulva cancer in Denmark. BMC Public Health. 2012; 17(1082):1-7.

25. Olsen J, Jorgensen TR, Rubek N. The economic burden of head and neck cancers in Denmark. Int J Otolaryngol Head Neck Surg. 2013;2:103-9.

26. Statens Serum Institut. The Cancer Register. Statistics and analyses [Cancerregisteret. Tal og analyse]. Copenhagen. 2012.

27. National Board of Health. Reduction in the risk of cervical cancer by vaccination against human papillomavirus (HPV) - a health technology assessment. Copenhagen: National Board of Health, Danish Centre for Health Technology Assessment; 2007 Jan.

28. Usher C, Tilson L, Olsen J, Jepsen M, Walsh C, Barry M. Cost-effectiveness of human papillomavirus vaccine in reducing the risk of cervical cancer in 
Ireland due to HPV types 16 and 18 using a transmission dynamic model. Vaccine. 2008;26:5654-61.

29. Jit M, Chapman R, Hughes O, Choi YH. Comparing bivalent and quadrivalent human papillomavirus vaccines: economic evaluation based on transmission model. BMJ. 2011;343:d5775.

30. Burchell AN, Richardson H, Mahmud SM, Trottier H, Tellier PP, Hanley J, et al. Modeling the sexual transmissibility of human papillomavirus infection using stochastic computer simulation and empirical data from a cohort study of young women in Montreal, Canada. Am J Epidemiol. 2006;163:534-43.

31. Sørensen J, Davidsen M, Gudex C, Pedersen KM, Bronnum-Hansen H. Danish EQ-5D population norms. Scand J Public Health. 2009;37:467-74.

32. Statens Serum Institut. EPI-news. Surveillance and prevention of infectious diseases. Revision of the HPV vaccination program for 12613 years old girls. 2014, week 28-32. [EPI-nyt. Overvågning og forebyggelse af smitsomme sygdomme. Anndring af HPV-vaccinationsprogrammet for 12- og 13-årige piger. 2014, Uge 28-32]. Copenhagen. 2014.

33. Statens Serum Institut. EPI-news. Surveillance and prevention of infectious diseases. HPV vaccination - vaccination coverage 2012. Week 20 - 2013 [EPI-NYT. Overvågning og forebyggelse af smitsomme sygdomme. HPV-vaccination - tilslutning 2012. Uge 20 - 2013]. Copenhagen. 2013.

34. Australian Government. National Immunisation Program Schedule [Internet]. http://www.health.gov.au/internet/immunise/publishing.nsf/ Content/4CB920F0D49C61F1CA257B2600828523/\$File/nip-schedule-card-hibmenc-update.pdf. 2013. Available from: http://www.health.gov.au/ internet/immunise/publishing.nsf/Content/4CB920F0D49C61F1CA257B2 600828523/\$File/nip-schedule-card-hib-menc-update.pdf

35. Kjaer SK, Tran TN, Sparen P, Tryggvadottir L, Munk C, Dasbach E, et al. The burden of genital warts: a study of nearly 70,000 women from the general female population in the 4 Nordic countries. J Infect Dis. 2007;196:1447-54.

\section{Submit your next manuscript to BioMed Central and take full advantage of:}

- Convenient online submission

- Thorough peer review

- No space constraints or color figure charges

- Immediate publication on acceptance

- Inclusion in PubMed, CAS, Scopus and Google Scholar

- Research which is freely available for redistribution 